\title{
Pilot study of submucosal radiofrequency for epistaxis in hereditary hemorrhagic telangiectasia*
}

\section{G. Mortuaire' , O. Boute², P.Y. Hatron³ , D. Chevalier}

' Department of Otorhinolaryngology and Head and Neck surgery, Huriez Hospital, University of Lille, France

2 Department of Medical Genetics, Jeanne de Flandre Hospital, University of Lille, France

${ }^{3}$ Department of Internal Medicine, Huriez Hospital, University of Lille, France
Rhinology 51: 355-360, 2013

DOI:10.4193/Rhino13.027

*Received for publication:

April 2, 2013

Accepted: July 13, 2013

\section{Summary}

Objective: To assess the safety and efficacy of submucosal radiofrequency (RF) treatment for hereditary hemorrhagic telangiectasia $(\mathrm{HHT})$ with mild or moderate epistaxis.

Methodology: We carried out a prospective pilot study of 16 consecutive patients with HHT-related epistaxis from June 2010 to April 2012. Under local anesthesia, RF was applied to one or both sides of the nose from the columella beneath the septal mucosal (50 joules per puncture). Patients were sent a questionnaire at least six months after the procedure.

Results: RF under local anesthesia was well tolerated, according to visual analog scale scores. Neither crusting nor pain was reported one week after the intervention. The frequency of epistaxis per day and per month was significantly lower after RF. The duration of bleeding also decreased from more than 10 minutes to less than 5 minutes in two thirds of patients. Thirteen of the 16 patients were satisfied with the technique and would request it for subsequent procedures to treat repeated bleeding.

Conclusion: Submucosal RF treatment for HHT is a safe, well tolerated procedure with significant efficacy in the short term. It should be considered as an alternative technique for managing HHT-related epistaxis, although long-term results remain to be evaluated.

Key words: epistaxis, hereditary hemorrhagic telangiectasia, radiofrequency, treatment technique

\section{Introduction}

Hereditary hemorrhagic telangiectasia (HHT) or Osler-WeberRendu syndrome is a rare disease displaying autosomal dominant inheritance and affecting approximately 1 in 6,000 people (1). It is characterized by mucocutaneous telangiectasia with recurrent, spontaneous epistaxis. It may cause severe anemia, requiring intravenous iron and blood transfusions, and impairing quality of life in many cases ${ }^{(2)}$. Diverse medical and surgical treatment options have been proposed for the control of HHTassociated epistaxis.

In severe cases, with daily epistaxis lasting more than $30 \mathrm{~min}$ and frequent transfusions, local surgery (packing, electrocoagulation, instillation of a sclerozing agent, embolization, septal dermoplasty) has been shown to be of variable efficacy ${ }^{(3)}$. Drugbased treatments (tranexamic acid, hormone therapy) have also been proposed with a good response on bleeding scores in a recent study of daily delivery of an anti-oestrogenic agent (tamoxifen) ${ }^{(4)}$. Intranasal treatment with an antiangiogenic agent (bevacizumab) has recently been shown to control epistaxis effectively ${ }^{(5,6)}$. In cases of intractable nasal bleeding, a procedure with complete nostril closure has been proposed. This approach gives satisfactory results but also causes certain difficulties due to nasal obstruction ${ }^{(7)}$.

For mild (a few episodes of epistaxis/week) and moderate (1-2 times/day and less than 10 transfusions over the patient's 
lifetime) cases, local surgery, including techniques such as argon laser therapy, are suitable ${ }^{(8)}$. Nasal crusting due to direct mucosal damage, with persistent bleeding immediately after the intervention, is often reported in the postoperative period.

We describe here an alternative method, based on submucosal radiofrequency (RF) treatment, for achieving submucosal sclerosis of nasal telangiectasia. This procedure is often performed during volumetric tissue reduction in cases of inferior turbinate hypertrophy in patients with chronic rhinitis ${ }^{(9)}$. RF treatment preserves the overlying mucosa and causes only minor postoperative discomfort ${ }^{(9)}$. The use of this treatment in HHT patients has not been investigated before.

The purpose of this pilot study was to evaluate a submucosal septal RF procedure for treating mild and moderate epistaxis in patients with $\mathrm{HHT}$.

\section{Materials and methods}

\section{Patients}

We carried out a prospective pilot study from June 2010 to April 2012 on 16 consecutive adult patients with $\mathrm{HHT}$ referred to our department for persistent nasal bleeding. In each case, unilateral or bilateral submucosal RF treatment of nasal mucosa telangiectasia was proposed. Clinical features of HHT-related diseases of the lung, liver, central nervous system and gastrointestinal tract were investigated. Preoperative hemoglobin concentration ( $\mathrm{g} / \mathrm{dl})$ and concomitant medical treatments were noted. Each patient gave informed consent for data collection and mining. A questionnaire was completed before surgery, to evaluate the severity of epistaxis (number of episodes per day and per month, duration of bleeding).

\section{Surgical technique}

Each procedure was conducted under local anesthesia, without sedation, in a day-care unit. Gentle nasal packing was performed for 15 minutes, with a piece of gauze, $5 \mathrm{~cm}$ long and $1 \mathrm{~cm}$ wide, soaked in a solution of lidocaine hydrochloride $(5 \mathrm{~g} / 100 \mathrm{ml})$ and naphazoline $(20 \mathrm{mg} / 100 \mathrm{ml})$. We then injected $5 \mathrm{ml}$ of a solution of lidocaine hydrochloride and epinephrine $(10 \mathrm{mg} / \mathrm{ml})$ via a 25-gauge spinal needle from the columella beneath the anterior third of the septal mucosa covered with telangiectasias (Figure 1). We monitored blood pressure and oxygen saturation, with a pulse oximeter, throughout the procedure.

RF treatment was carried out endoscopically, with a 30-degree rigid nasal fiberoptic endoscope. The Ellman Surgitron ${ }^{\circledR} 4.0$ Dual RF/120 IEC (Ellman Surgitron FFPF EMC Radiosurgical Device, Ellmann International, New York, USA) portable unit was used. Three submucosal punctures were made in the columella, in one or both nostrils with the IEC-16D bipolar handpiece (Figure $2 A$ ). In coagulation mode, we applied 50 joules (10 watts over a period of $5 \mathrm{sec}$ ) to each puncture, to "whiten" the telangiectasias and surrounding nasal mucosa (Figure 2B). Any bleeding during the procedure was treated by additional lidocaine/naphazoline packing, without direct cauterization of the mucosa. No nasal dressing was required after the procedure. The patients were discharged from the day-care unit after one hour of medical supervision.

\section{Questionnaire}

A questionnaire was sent to each patient at least six months later. They were asked to evaluate pain and crusting in the first week after the intervention, on a visual analog scale running from 0 to 10 (with 10 corresponding to worst pain and crusting bearable), and to quantify the current frequency and duration of episodes of epistaxis. Any additional procedures performed were also noted, including subsequent RF treatment on the contralateral side in cases of initially unilateral surgery, in particular.

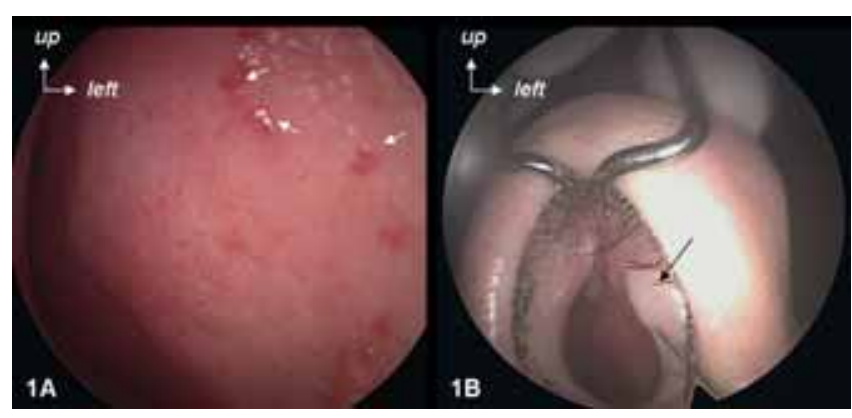

Figure 1. Endoscopic view (30-degree telescope) of septal telangiectasias in a right nasal fossa. (A) Telangiectasias are visible (white arrows) in the anterior part of the septum. (B)The 25-gauge spinal needle is inserted into the columella area (black arrow) beneath the septal mucosa. Vasoconstriction and local anesthesia are achieved with lidocaine hydrochloride and epinephrine $(10 \mathrm{mg} / \mathrm{ml})$.

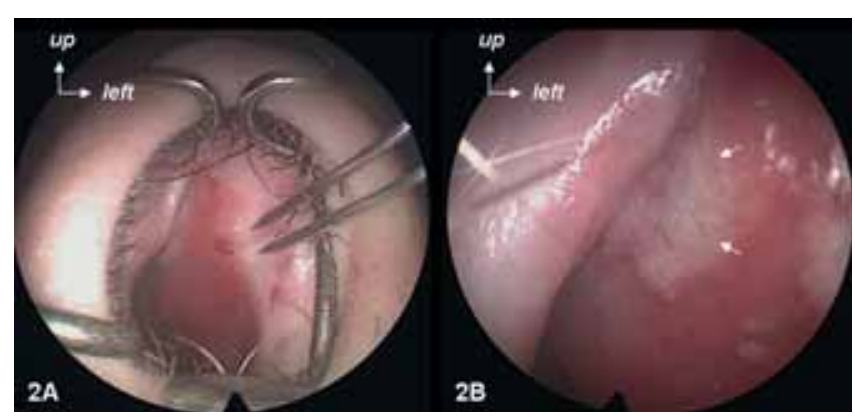

Figure 2. Introduction of the bipolar radiofrequency handpiece and endoscopic view (30-degree telescope) of the local effect of radiofrequency in a right nasal fossa.

(A) The bipolar handpiece is inserted via the intersepto-columellar space, to provoke controlled submucosal coagulation of the telangiectasias. (B) A whitening of the surrounding mucosa is observed (white arrows). 


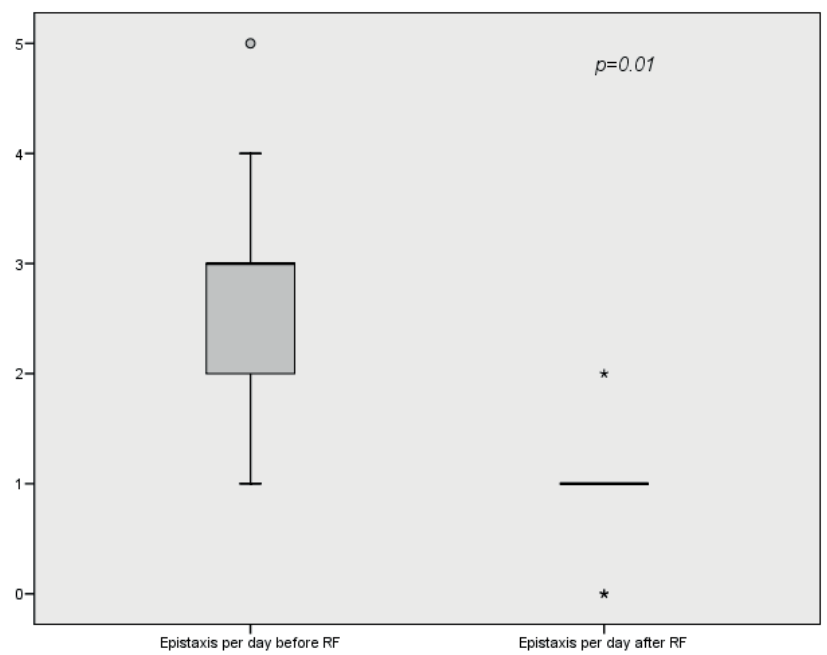

Figure 3. Frequency of epistaxis per day, before and after radiofrequency treatment. A comparison of the number of events per day before (mean: 2.7) and after RF treatment (mean: 0.9) revealed a significant decrease in the frequency of events after treatment $(p=0.01)$.

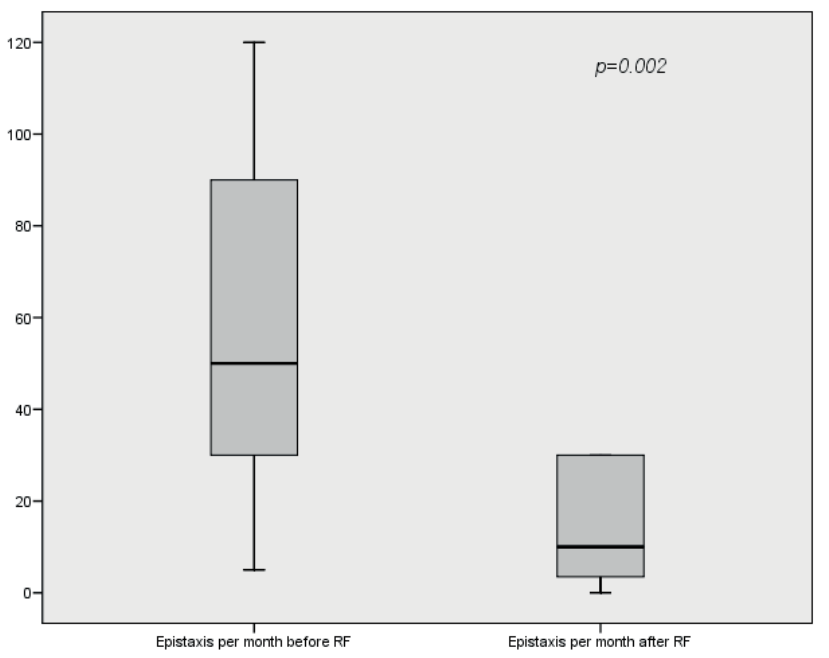

Figure 4. Frequency of epistaxis per month, before and after radiofrequency. The numbers of events per month before (mean: 57.3) and after RF treatment (mean: 14.1) were compared and a significant decrease in the frequency of events after treatment was observed $(p=0.002)$.

\section{Statistical methods}

Data were input into Microsoft Excel ${ }^{\circledR}$ and statistical analysis was performed with SPSS ${ }^{\circledR} 15.0$ software. The non parametric Wilcoxon test was used to compare the means of paired samples. Values of $p<0.05$ were considered significant.

\section{Results}

All 16 patients completed all the items of the pre- and postoperative questionnaires. There were 10 women and six men, with a mean age of 48 years (range: 25 to 61 years). All patients had suffered epistaxis since childhood, but the definitive diagnosis of $\mathrm{HHT}$, according to the Curacao diagnostic criteria, was established between 2003 and 2011. A complete disease assessment was carried out for each patient. Cutaneous telangiectasias were observed in 14 cases (87\%). Gastrointestinal lesions were detected by endoscopy in three cases (19\%). CT scans of the liver revealed abnormalities in four cases (25\%). Lung CT scans revealed arteriovenous malformations in five cases (31\%). Brain lesions were identified on CT scans in two cases (12\%). Mean hemoglobin concentration was 11.6 (range: 6.4 to 15.6), with blood transfusion required in one case and iron supplements prescribed in seven cases (43\%).

Before their referral to our institution, 10 of the patients (62\%) had already been treated surgically for nose bleeds: by cauterization with electrocoagulation in eight cases, mucosal cauterization with a laser in one case and acrylic polymer scleroembolization in one case. Five patients (31\%) had received drug treatments: tranexamic acid alone in three cases, tranexamic acid + thalidomide in one case, and tranexamic acid + dydrogesterone in one case.
In preoperative assessments of nasal bleeding, the mean frequency of epistaxis was 2.7 events per day and 57.3 events per month (Figures 3 and 4), with considerable day-to-day variation. Bleedings events lasted for between $30 \mathrm{sec}$ and $5 \mathrm{~min}$ in two cases, between $5 \mathrm{~min}$ and $10 \mathrm{~min}$ in four cases and for more than 10 min in 10 cases (Figure 5). RF treatment was unilateral in 12 cases, and bilateral in the other four cases. Bilateral surgery was proposed in cases in which epistaxis was equally frequent on both sides of the nose, with visible telangiectasias on both sides. During the procedure, the mean level of pain on visual analog scale was $4.7 / 10$. In the first week after the intervention, mean crusting score was $6 / 10$ and the persistent pain score was $2.6 / 10$. No pain or crusting was reported after the first postoperative week.

The postoperative questionnaire was completed a mean of 14 months after the RF procedure. Mean follow-up was 16 months (from 6 to 30 months). Epistaxis recurred in 12 patients (75\%), with a bleeding-free period of one week in four cases, and one month in the other eight cases. Postoperative assessments of nasal bleeding showed a mean frequency of 0.9 events per day and 14.1 events per month (Figures 3 and 4).The mean duration of each event was less than $30 \mathrm{sec}$ in six cases, between $30 \mathrm{sec}$ and $5 \mathrm{~min}$ in five cases, between $5 \mathrm{~min}$ and $10 \mathrm{~min}$ in three cases and more than $10 \mathrm{~min}$ in two cases (Figure 5). We observed a general decrease in the duration of epistaxis after RF treatment in most patients, with only three patients showing no improvement for this variable. None of the patients reported a worsening of symptoms after RF treatment (Figure 6). The numbers of epistaxis events per day and per month were significantly lower 


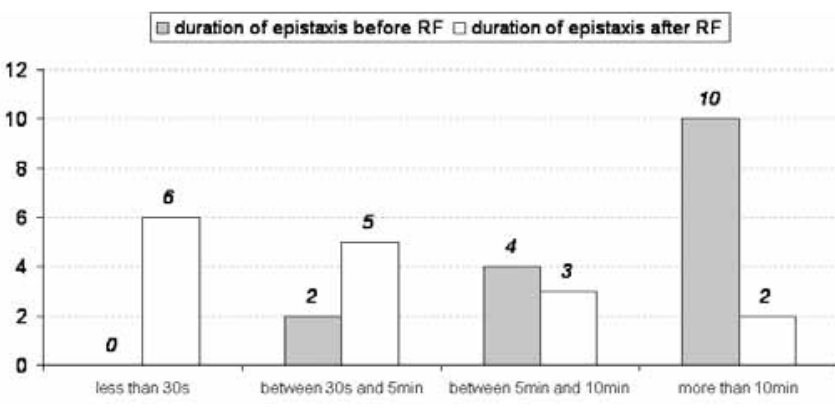

Figure 5. Comparison of the duration of the epistaxis before and after radiofrequency treatment. We observed an overall decrease in the duration of the epistaxis after RF treatment.

after RF treatment $(p=0.01$ and $p=0.002$, respectively. Figures 3 and 4).

The patients were asked whether they would recommend this treatment to other patients with HHT-related telangiectasias. Responses were anonymous, and 13 of the 16 patients said that they would recommend this procedure to others and would be willing to repeat the procedure themselves if bleeding recurred. In three of the 12 cases initially undergoing unilateral treatment, a staged approach was applied with a second procedure carried out on the contralateral side four to six weeks after the initial intervention.

\section{Discussion}

Epistaxis is the most common and bothersome presenting symptom in patients with HHT. In a psychosocial study of 115 HHT patients, frequent episodes of epistaxis and abundant bleeding have been described as deeply impairing the quality of life ${ }^{(10)}$. The management of HHT-related epistaxis has always proved challenging to otolaryngologists. No particular procedure has yet been validated in the long term and the approach used often depends on local guidelines and institutional habits. As pointed out by Lund et al., treatment algorithms are based on the frequency and severity of bleeding events ${ }^{(8)}$. This has resulted in the development of several different grading systems for assessing epistaxis in $\mathrm{HHT}^{(11)}$. As a result, it is difficult to compare and evaluate the effectiveness of different types of treatment for epistaxis evaluated with different scales.

There are several drug-based treatments, and surgical options range from mucosal cauterization to nostril closure. Various devices (cryocauters, chemocauters, electrocauters, lasers therapy, Coblator $^{\circledR}$ ) may be used for the initial treatment ${ }^{(12)}$. However, their effects last for only a short period. Immediate bleeding, persistent crusting and septal perforations are frequently described. The intramucosal injection of fibrogenic and

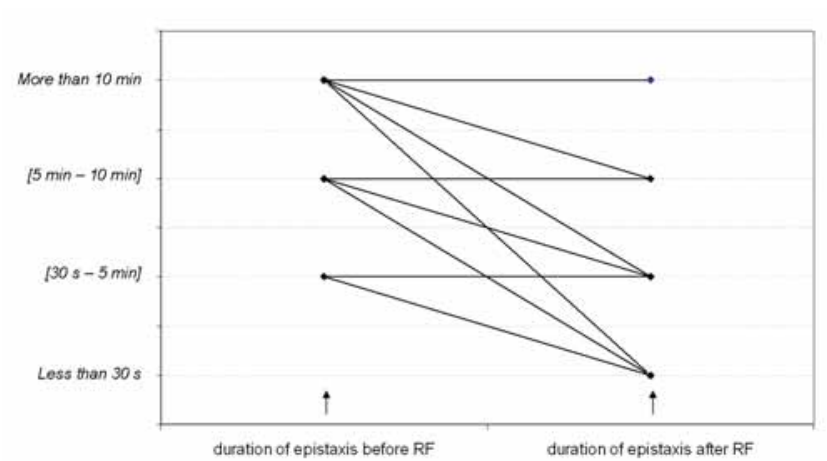

Figure 6. Duration of epistaxis before and after radiofrequency treatment, for each patient. Thirteen patients reported a decrease in the duration of the epistaxis. The other three patients reported no improvement. No patient reported a worsening of epistaxis duration after RF.

thrombogenic substances has proved effective. In a series of 300 injections of polydocanol on 45 patients, Morais et al. reported an overall improvement of bleeding with respect to frequency and quantity ${ }^{(13)}$. This procedure requires specific skills to ensure that the injection is performed correctly, given the risk of neuroophthalmologic side effects ${ }^{(14)}$. Nasal septodermoplasty has also been used, but may be a difficult technique to perform. Graft failure with recurrent epistaxis can occur ${ }^{(15)}$. In cases of intractable epistaxis, resulting in a need of frequent transfusions, a radical approach based on nasal closure has been used by some authors, and has been shown to be effective ${ }^{(7,12)}$. In these cases, epistaxis can be prevented by completely preventing airflow through the treated nostril. Nasal closure procedure is the only long-lasting effective treatment reported to date.

With a view to finding a simple way of managing epistaxis in mild or moderate cases, we investigated the use of RF treatment to achieve the submucosal sclerosis of nasal septum telangiectasias. This approach is routinely used in our institution for the treatment of hypertrophic rhinitis and its safety has been clearly demonstrated ${ }^{(9)}$. RF treatment is widespread in clinical practice. Standard electrocautery devices require temperatures of up to $800^{\circ} \mathrm{C}$, causing immediate mucosal disruption and coagulation necrosis, whereas treatment with RF devices results in qualitatively greater preservation of the normal sinonasal mucosa at temperatures of between 60 and $90^{\circ} \mathrm{C}^{(16,17)}$. Collateral damage to surrounding tissues is limited by the lower operating temperature achieved by the precise regulation of energy delivery from the device ${ }^{(17)}$. RF treatment of the nasal turbinate induces the coagulation of venous sinusoids and of loose connective tissue, leading to submucosal fibrosis ${ }^{(18)}$. Based on these effects, we decided to test RF treatment via a septal submucosal approach as a possible treatment for HHT-related epistaxis.

This prospective pilot study demonstrated the safety and the efficacy of the submucosal procedure for decreasing the frequen- 
cy and duration of nose bleeds. This procedure has never before been described for the treatment of this condition. It made it possible to treat nasal HHT telangiectasias in a day hospital without inducing immediate and severe epistaxis. Overall, this procedure was well tolerated with 13 of the 16 patients giving it their approval.

Crusting was observed during the postoperative period, despite the use of a submucosal approach. When RF is applied through the inferior turbinates in hypertrophic rhinitis, the energy is delivered deep inside the tissue, with little damage of the turbinal mucosa, thus limiting the formation of postoperative crusts. The submucosal space is thinner in the septal framework. Mucosal injury was minimized by decreasing the power setting to $10 \mathrm{~W}$ for each $5 \mathrm{sec}$ application (our power setting for inferior turbinoplasty is $40 \mathrm{~W}$ applied for $20 \mathrm{sec}$ on each side). Moreover, the use of an endoscope to view mucosal whitening directly made it possible for the surgeon to stop the procedure at precisely the right time. With this high-precision approach, no case of septal perforation was observed, despite the bilateral application of RF treatment in four patients.

The small size of our population and the relatively short mean follow-up of 16 months may have had an impact on the results obtained and their statistical value. Only four patients with initially mild disease remained free from epistaxis during the postoperative period. Recurrence is likely to occur in these four patients and bleeding events will probably worsen in the other 12 patients. However, submucosal RF treatment can be considered a valid alternative procedure for the management of the HHT-related epistaxis. Given its safety, this approach can be used for procedures on one or both sides of the nose, depending on the patient's symptoms and wishes. Even if RF treatment was not applied on the lateral nasal wall in our study, this procedure should also be considered for telangiectasias on the turbinate mucosal area.

A new targeted drug-based treatment with bevacizumab has recently been evaluated. Bevacizumab is a recombinant humanized monoclonal antibody that inhibits the biological activity of VEGF, thereby preventing endothelial cell proliferation and angiogenesis ${ }^{(19)}$. This drug has been administrated intranasally at various doses, via several different techniques ${ }^{(5,6,19,20)}$. The results obtained suggest that bevacizumab is a promising new treatment for HHT-associated epistaxis. However, this treatment requires further evaluation in the long term focusing on the appropriate indications and the potential late side effects of repeated anti-angiogenic drug treatments. A single bevacizumab injection currently costs more than most of the "classic" types of treatment ${ }^{(20)}$. Local surgical approaches, such as submucosal septal RF treatment, deserve further consideration in the treatment of HHT. RF treatment appears to be an interesting option for patients with mild or moderate epistaxis, although its longterms effects have yet to be assessed (for instance, occurrence of septal perforation in recurrent bilateral treatment). Additional studies are required before any firm conclusions can be drawn.

\section{Concluding remarks}

HHT-related epistaxis is a condition of major concern to otolaryngologists. No surgical or drug-based treatment has yet been shown to decrease nasal bleeding in the longer term. Multiple surgical procedures have been described from electrocauterization to nostril closure. We propose here a non invasive technique based on the submucosal radiofrequency treatment of the septal telangiectasias under local anesthesia. This approach seems to be safe and effective in the short term, for the treatment of mild and moderate epistaxis. As RF treatment limits mucosal injury, this technique can be reapplied, to one or both sides of the nose, if bleeding is subsequently aggravated. The patients expressed satisfaction with the procedure, encouraging us to pursue our evaluation of its mid and long-term effects. $\mathrm{RF}$ should be considered a potential tool among the complex arsenal of treatments available for HHT telangiectasia. However, other local procedures, including promising new treatments, such as local bevacizumab in particular, should not be neglected either.

\section{Authorship contribution}

GM: study design, data acquisition, writing and statistical analysis; OB: data collection; PYH: data collection; DC: senior reviewer.

\section{Conflict of interest}

None to declare.

\section{References}

1. Shovlin CL, Guttmacher AE, Buscarini E, et al. Diagnostic criteria for hereditary hemorrhagic telangiectasia (Rendu-Osler-Weber syndrome). Am J Med Genet. 2000; 91: 66-67.

2. Olitsky SE. Topical timolol for the treatment of epistaxis in hereditary hemorrhagic telangiectasia. Am J Otolaryngol. 2012; 33 375-376.

3. Samson G, García de la Calera A, DupuisGirod S, et al. Ex vivo study of bevacizumab transport through porcine nasal mucosa. Eur J Pharm Biopharm. 2012; 80: 465-469.

4. Yaniv E, Preis M, Shevro J, Nageris B, Hadar T. Anti-estrogen therapy for hereditary hemorrhagic telangiectasia - a long-term clinical trial. Rhinology. 2011; 49: 214-216.

5. Chen S, Karnezis T, Davidson TM. Safety of intranasal bevacizumab (Avastin) treatment in patients with hereditary hemorrhagic telangiectasia-associated epistaxis. Laryngoscope. 2011; 121: 644-646.

6. Brinkerhoff BT, Choong NW, Treisman JS,
Poetker DM. Intravenous and topical intranasal bevacizumab (Avastin) in hereditary hemorrhagic telangiectasia. Am J Otolaryngol. 2012; 33: 349-351.

7. 7. Lund VJ, Howard DJ. Closure of the nasal cavities in the treatment of refractory hereditary haemorrhagic telangiectasia. J Laryngol Otol. 1997; 111: 30-33.

8. Lund VJ, Howard DJ. A treatment algorithm for the management of epistaxis in hereditary hemorrhagic telangiectasia. Am J Rhinol. 1999; 13: 319-322. 
9. Lin $H C$, Lin PW, Friedman $M$, et al. Long term results of radiofrequency turbinoplasty for allergic rhinitis refractory to medical therapy. Arch Otolaryngol Head Neck Surg. 2010; 136: 892-895.

10. Loaëc $M$, Morinière $S$, Hitier $M$, Ferrant $O$, Plauchu $\mathrm{H}$, Babin E. Psychosocial quality of life in hereditary haemorrhagic telangiectasia patients. Rhinology. 2011; 49: 164-167.

11. Al-Deen S, Bachmann-Harildstad G. A grading scale for epistaxis in hereditary haemorrhagic teleangectasia. Rhinology. 2008; 46: 281-284.

12. Ichimura K, Kikuchi H, Imayoshi S, Yamauch $\mathrm{T}$, Ishikawa K. Are patients with severe epistaxis caused by hereditary hemorrhagic telangiectasia satisfied with nostril closure surgery? Auris Nasus Larynx. 2012; 39: 59-64.

13. Morais D, Millás T, Zarrabeitia R, Botella LM, Almaraz A. Local sclerotherapy with polydocanol (Aethoxysklerol ${ }^{\oplus}$ ) for the treatment of Epistaxis in Rendu-Osler-Weber or Hereditary Hemorrhagic Telangiectasia (HHT): 15 years of experience. Rhinology. 2012; 50: 80-86
14. Borsik M, Herbreteau D, Deffrennes D, et al [Treatment of epistaxis in Rendu-Osler disease by intramucosal injection of ethibloc] Ann Otolaryngol Chir Cervicofac. 1992; 109: 273-276.

15. Ichimura K, Kikuchi H, Imayoshi S. A new method of nasal dermoplasty (MW grafting method) in patients with large septal perforation. Laryngoscope. 2011; 121: 1715-1717.

16. Farmer SE, Eccles R. Understanding submucosal electrosurgery for the treatment of nasal turbinate enlargement. J Laryngol Otol. 2007; 121: 615-622.

17. Kakarala K, Faquin WC, Cunningham MJ Radiofrequency volumetric tissue reduction of the inferior turbinate in a sheep model. Laryngoscope. 2012; 122: 724-729.

18. Gouveris H, Nousia C, Giatromanolaki A et al. Inferior nasal turbinate wound healing after submucosal radiofrequency tissue ablation and monopolar electrocautery: histologic study in a sheep model. Laryngoscope. 2010; 120: 1453-1459.

19. Simonds J, Miller F, Mandel J, Davidson TM The effect of bevacizumab (Avastin) treatment on epistaxis in hereditary hemorrhag- ic telangiectasia. Laryngoscope. 2009; 119 988-992.

20. Dheyauldeen S, Østertun Geirdal A, Osnes T, Vartdal LS, Dollner R. Bevacizumab in hereditary hemorrhagic telangiectasia-associated epistaxis: effectiveness of an injection protocol based on the vascular anatomy of the nose. Laryngoscope. 2012; 122: 1210-1214.

\section{Geoffrey Mortuaire}

\section{Service d'ORL de chirurgie}

cervico-faciale

Hôpital Huriez

CHRU Lille

59000 France

Tel: +33-32-044 5675

Fax: +33-32-044 6220

E-mail: g-mortuaire@chru-lille.fr

\section{1th INTERNATIONAL COURSE IN ADVANCED SINUS SURGERY TECHNIQUES}

Dissection course with fresh frozen cadaver heads

Teacher of Honour:

Robert C Kern, MD

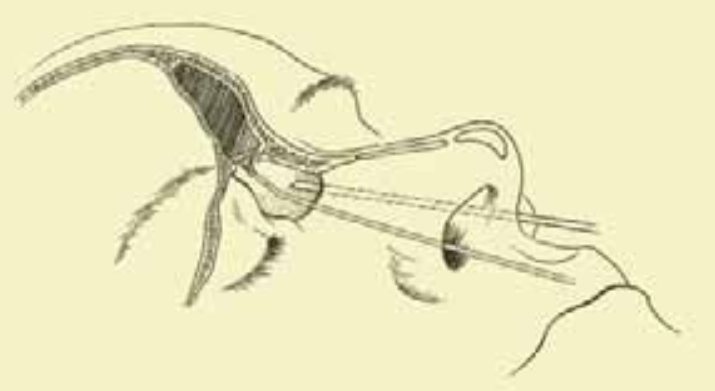

March 27-28, 2014

Department of Otorhinolaryngology

Academic Medical Center of the University of Amsterdam

The Netherlands

For further information contact Wytske J. Fokkens, $M D, P h D$ ENT dept. AMC Course Secretariat

Tel: 00312056685 86 / Fax 0031205669573

Email:m.b.vanhuiden@amc.uva.nl 